\title{
Research on the Mechanism of Chuanxiong in Treating Diabetic Nephropathy by Network Pharmacology and Molecular Docking Technique
}

\section{Changyan Li}

Kunming Medical University First Affilliated Hospital

\section{Feng Su}

Kunming Medical University First Affilliated Hospital

\section{Le Zhang}

University of California Riverside

Fang Liu

Sichuan University West China Hospital

\section{Zhen Li}

Kunming Medical University First Affilliated Hospital

Wenxing Fan ( $\nabla$ fanwx2020@163.com )

Kunming Medical University First Affilliated Hospital

Jing Yang

Kunming Medical University First Affilliated Hospital

\section{Niroj Mali}

Kunming Medical University First Affilliated Hospital

\section{Research Article}

Keywords: chuanxiong, diabetic nephropathy, network pharmacology, molecular docking

Posted Date: November 23rd, 2021

DOI: https://doi.org/10.21203/rs.3.rs-1094538/v1

License: (c) (1) This work is licensed under a Creative Commons Attribution 4.0 International License. Read Full License 


\section{Abstract \\ Background}

Chuanxiong hort. It has been used in the clinic, but its main active components and drug mechanism are unknown.

\section{Objective}

To explore the mechanism of diabetic nephropathy (DN) treated with chuanxiong hort.

\section{Methods}

the active components and targets of chuanxiong Hort. were collected by TCPSP database, the DN gene expression data were collected by NCBI database, DN related genes were obtained by differential analysis. Furthermore, String and Cytoscape tools were used to construct the regulatory network of chuanxiong and the main active components for DN treatment. At the same time, PPI network interaction analysis was performed for core genes and GO. KEGG analysis was performed, prediction of molecular docking using Auto Dock Tools for critical genes.

\section{Results}

eight active components of chuanxiong Hort. were screened out, and one was recorded repeatedly. They were Angelica lactone A, ligusticum chuanxiong naphthofurolactone, Myricetin, 1-palmitic acid-2-linoleic acid-3-olein and ferulic acid ester of conifer. In addition, the active components interact with 15 disease targets, namely PGR, NR3C1, NCOA1, NCOA2, NR3C2, PTGS1, F2, F7, ESR1, AR, PPARG, ESR2, DPP4, HSP90AA1 and PRKACA. Besides, GO analysis of target genes, involved in the regulation of DN by chuanxiong Hort., were the regulation of cellular response to steroid hormone stimulation, the binding of nuclear receptors to steroid hormone receptors and the principle of cAMP-dependent protein kinase complexes. What's more, results of KEGG analysis showed that estrogen signalling pathway, endocrine and other factors-regulated calcium reabsorption and adipocyte adipogenesis played a role in the regulation of DN. Further molecular docking showed that NCOA1 and NCOA2 could interact with Angelica lactone A, Myricetin, Chrysophanol, chuanxiong naphthalize and Chrysophanol.

\section{Conclusion}

the active components of chuanxiong Hort., including Angelica Lactone A, Myricetin, Chrysophanol, chuanxiong naphthafunolide and Chrysophanol, can affect the regulation of estrogen signalling pathway, endocrine and other factors regulating calcium reabsorption and adipogenesis of adipocytes through the 
regulation of steroid hormone stimulation and regulation of cAMP-dependent protein kinase complex, and then play a therapeutic role on DN.

\section{Introduction}

Chronic kidney disease caused by diabetes is called diabetic nephropathy (DN). DN is a chronic microvascular disease [1]. Recent studies have shown that the prevalence of DN in adults with diabetes varies by region: $27.9 \%$ in Spain, $32.4 \%$ and $42.3 \%$ in the United Kingdom, $47.0 \%$ in France and $63.9 \%$ in Shanghai [2]. DN is a common and underrated complication of diabetes mellitus. If we don't intervene early, it will bring more complications and bad results to the patient. Although some drugs have been tested in animal models and can play a role [3-6], so far, due to the complex pathophysiological mechanism of DN, there is no specific drug treatment. Traditional Western drugs such as ACEI/ARB are effective [7, 8], but there is still a treatment blind spot. Traditional Chinese medicine is rich in resources, has great potential to explore the active components of Chinese medicine as the main or auxiliary medication to improve the prognosis of DN patients.

Chuanxiong, as traditional Chinese medicine, is the rhizome of ligusticum chuanxiong (Umbelliferae). The active components of chuanxiong mainly include phenolphthalein, organic phenolic acids and alkaloids [9-11]. A clinical parallel control study on the treatment of DN with chuanxiong injection combined with Puli showed a significant improvement in $24 \mathrm{~h}$ urinary protein levels, serum creatinine and serum urea nitrogen in DN patients; this study suggests that chuanxiong Hort. may be used as an auxiliary drug for the effective treatment of DN progression [12]. On the premise of having a definite curative effect as clinical medication, studying the specific active components of chuanxiong and making use of them will exert the best corrective influence on DN.

In recent years, internet pharmacology has contributed to discovering practical drug active components in traditional Chinese medicine. In addition, the prediction of the action target of active drug components has also shorted the time for exploring the mechanism of traditional Chinese medicine; It is urgent to find out the practical active components of chuanxiong Hort in the treatment of DN by means of network pharmacology and molecular docking technique.

\section{Data And Methods}

\subsection{Data sets of active components and DN gene of chuanxiong Hort.}

the search term "chuanxiong" was used to obtain the active components and target sites of chuanxiong Hort. According to Lipinski's five rules[13], by using relative molecular mass $(\mathrm{MW})<500$, hydrogen bond donor (Hendon) $\leq 5$, hydrogen bond acceptor $(H A C C) \leq 10$, lipid-water partition coefficient $P<5.0$ and pharmacokinetics index drug similarity $(D L) \geq 0.18$ as the screening conditions. At the same time, the target information was annotated with Uniprot (https://www.Uniprot.org/) database. GSE142025 gene 
expression data set of DN was used to analyze the differences of GSE142025 from GEO (9 control samples and $27 \mathrm{DN}$ samples based on the "Limma" R package). Under the condition of LogFoldchange = 1 , adjust $P=0.05$, the $\mathrm{DN}$-related genes were obtained.

\subsection{Construction of a regulatory network of DN and active components of chuanxiong Hort.}

Veen analysis of DNA differential gene and active component gene of chuanxiong was carried out, and the core gene-regulating $\mathrm{DN}$ of chuanxiong was obtained. Cytoscape 3.7.2 software was used to visualize the regulatory network.

\subsection{Construction of target protein interaction network mediated by core target genes.}

The active chemical components of chuanxiong were analyzed by the String database(https://string$\mathrm{db}$. org/), and the results were optimized by Cytoscape 3.7.2 software.

\subsection{GO and KEGG Pathway Enrichment Analysis}

Gene Ontology (GO)(http://geneontology.org/) is a database established by Gene Ontology, which is used to screen proteome based on $P<0.05$. Go analysis can be divided into three categories: molecular function (MF), biological process (BP) and cell composition (CC); through them, the function of genes can be defined and described in many ways. Based on the annotation co-degree, the annotation items are clustered, and the clustering values are given by using the module and clustering algorithm. The higher the score, the more prominent the gene is on the gene list. In addition, Kyoto Encyclopedia of Genes and Genomes (KEGG) (https://www.genome.jp/kegg/) is a comprehensive database that integrates genomic, chemical and systemic functional information on the genetic pathways of different species. Use the cluster profile R package "Org.hs.eg.db". Screening protein functional groups in KEGG annotation with $P<$ 0.05. The biological functions of autophagic genes and the biological pathways involved were studied by enrichment analysis of available items. GO, and KEGG analysis was used in this study, and the setting was $P<0.05$.

\subsection{Macromolecular docking}

In order to study the effect of chuanxiong on DN, the key genes and the main active components of chuanxiong were screened and analyzed by molecular docking. The structure of active components of chuanxiong Hort was searched from Pubchem database(https://pubchem.ncbi.nlm.nih.gov/)and optimized by Chem3D software to minimize binding energy. In addition, the 3D structure of the core gene- 
mediated human protein was downloaded from the PDB database (https://www.ribs.org/), and the water and small molecular ligands of the protein structure were deleted by AutoDockTools, and the secondary structure was retained by hydrogenation, the range of active pocket for chemical structure adjustment of active components is introduced, and the suitable binding energy $\leq-5.0 \mathrm{~kJ} / \mathrm{mol}$ is selected to determine the result of molecular docking by using Vina software. Finally, PyMol2.5.1 software was used to optimize the docking results and select the optimal results.

\subsection{Main observational indicators}

To construct a target regulatory network of active components of chuanxiong- chuanxiong-disease-drug disease action, to directly reflect the regulatory effect of each active component, and to obtain the core gene regulatory relationship through the protein-protein interaction network mediated by target genes; GO, and KEGG were used to analyze the target regulation of active components and the mechanism of treating DN with chuanxiong. Molecular docking was used to predict the binding site and ability of Wallachia active components to target genes.

\section{Results}

\subsection{Main active components and target of chuanxiong Hort.}

main active components and action targets of chuanxiong were selected by Lipinski five rules and drug similarity, eight active components of chuanxiong were obtained, and one was recorded repeatedly, they are levitolide-a/levitolid-a, walllichilide, Myricanone, 1-palmitic-2-linoleic-3-olein (Plo), coniferylfcrate, Ethyl acrylate-7-aldehyde- $\beta$-carboline (1-beta-ethyl acrylate-7-aldehyde-beta-carboline), perolyrine and Chrysophanol are listed in Table 1, and the corresponding target information of 8 active components is listed in Table 2. 
Table 1

Basic information of active compounds of chuanxiong Hort.

\begin{tabular}{|lllllll|}
\hline Mol ID & Molecule Name & MW & AlogP & Hdon & Hacc & DL \\
\hline MOL002200 & Levistolide-A & 380.5 & 4.97 & 0 & 4 & 0.81739 \\
\hline MOL002102 & Levistolid A & 380.5 & 4.97 & 0 & 4 & 0.81738 \\
\hline MOL002157 & wallichilide & 412.6 & 4.823 & 0 & 5 & 0.70639 \\
\hline MOL002135 & Myricanone & 356.5 & 4.1 & 2 & 5 & 0.51262 \\
\hline MOL002141 & PLO & 316.5 & 3.485 & 1 & 2 & 0.43379 \\
\hline MOL002192 & Coniferylfcrulate & 356.4 & 3.637 & 2 & 6 & 0.39056 \\
\hline MOL002161 & 1-beta-ethylacrylate-7-aldehyde-beta- & 294.3 & 3.033 & 1 & 4 & 0.31157 \\
& carboline & 264.3 & 3.202 & 2 & 3 & 0.2747 \\
\hline MOL002140 & Perlolyrine & 254.3 & 2.76 & 2 & 4 & 0.2094 \\
\hline MOL001729 & Crysophanol & & & & & \\
\hline
\end{tabular}


Table 2

Basic information about the Targets of active compounds of chuanxiong Hort.

\begin{tabular}{|c|c|c|}
\hline Molld & MolName & Target \\
\hline \multirow[t]{3}{*}{ MOL002200 } & \multirow[t]{3}{*}{ Levistolide-A } & PGR \\
\hline & & NR3C1 \\
\hline & & NCOA1 \\
\hline MOL002192 & Coniferylfcrulate & ESR1 \\
\hline \multirow[t]{2}{*}{ MOL002161 } & \multirow{2}{*}{$\begin{array}{l}\text { 1-beta-ethylacrylate-7-aldehyde-beta- } \\
\text { carboline }\end{array}$} & PTGS1 \\
\hline & & HSP90AA1 \\
\hline \multirow[t]{3}{*}{ MOL002157 } & \multirow[t]{3}{*}{ Wallichilide } & NR3C2 \\
\hline & & NR3C1 \\
\hline & & NCOA2 \\
\hline \multirow[t]{4}{*}{ MOL002141 } & \multirow[t]{4}{*}{ PLO } & PGR \\
\hline & & NR3C2 \\
\hline & & NCOA2 \\
\hline & & NCOA1 \\
\hline \multirow[t]{2}{*}{ MOL002140 } & \multirow[t]{2}{*}{ Perlolyrine } & PRKACA \\
\hline & & F2 \\
\hline \multirow[t]{11}{*}{ MOL002135 } & \multirow[t]{11}{*}{ Myricanone } & PTGS1 \\
\hline & & PPARG \\
\hline & & NCOA1 \\
\hline & & HSP90AA1 \\
\hline & & GSK3B \\
\hline & & F7 \\
\hline & & F2 \\
\hline & & ESR2 \\
\hline & & ESR1 \\
\hline & & DPP4 \\
\hline & & CHEK1 \\
\hline
\end{tabular}




\begin{tabular}{|lll|}
\hline Molld & MolName & Target \\
\hline MOL002102 & Levistolid A & AR \\
& & PGR \\
& & NR3C1 \\
& & NCOA2 \\
MOL001729 & Crysophanol & NCOA1 \\
& & PTGS1 \\
& & PRKACA \\
& & NCOA2 \\
& & HSP90AA1 \\
\hline
\end{tabular}

\subsection{Potential therapeutic target of DN in chuanxiong rhizome.}

Gene expression data set GSE142025 was downloaded from the GEO database by the active components of chuanxiong Hort. and row differences of the data set were analyzed by using the "Limma" R program package under the conditions of Logfoldchange $=1$ and adjust $P=0.05$, a total of $3701 \mathrm{DN}$ differentially expressed genes (Figure 1A, B) were obtained, and 15 DN and chuanxiong were identified by Veen analysis (Figure 1C), there are also interactions between targeted regulatory gene-mediated proteins (Figure 1D).

\subsection{Target network of chuanxiong in the treatment of DN.}

Protein interaction analysis of core gene of ligusticum chuanxiong regulating $\mathrm{DN}$ was carried out through STRING database. Cytoscape 3.7.2 software was used to optimize the results, eight active components of Ligusticum chuanxiong Hort. ., including Angelica lactone a (Levistolide-A/Levistolid a), ligusticum chuanxiong (wallichuilide), Myricanone (Myricanone), 1-palmitic acid-2-linoleic acid-3-oleic acid glyceride (PLO), coniferylfcrate, were constructed, the regulatory model of 1-beta-Ethyl acrylate-7-aldehyde-betacarboline, Perolyrine and Chrysophanol on DN targets (Figure 2A), in which NR3C1, AR, ESR1, NCOA1, NCOA2 and PGR play key roles in the regulatory network (Figure 2B).

\subsection{Chuanxiong hort. It can affect steroid hormone signal transduction and activate the estrogen signal pathway to regulate DN.}


GO analysis and KEGG analysis of 15 potential target genes of chuanxiong Hort. regulating DN. The GO results suggest that the genes involved in the regulation of DN in chuanxiong are involved in the regulation of the cellular response to steroid hormone stimulation, the binding of steroid hormone receptors and the regulation of cAMP-dependent protein kinase complexes (Figure 3A); Besides, KEGG analysis showed that estrogen signalling pathway, endocrine and other factors regulating calcium reabsorption and adipogenesis (Figure 3B) played a role in the regulation of DN by chuanxiong Rhizome.

\subsection{NCOA1 and NCOA2 could interact with the active components of chuanxiong Hort.}

To further explore the regulatory role of chuanxiong on DN, the key genes and main active components of chuanxiong were screened and analyzed by molecular docking. After determining the range of the active pocket, we further use Vina software to predict the docking, select the appropriate binding energy, synthesize the number of hydrogen bond interactions, determine the result of molecular docking. Finally, Pymol2.5.1 software was used to optimize the docking results. The N224 and K220 groups of NCOA1 protein were selected to interact with the hydrogen bond of olide-a/Levistolid-a (Figure 4A, E). There are hydrogen bond interactions between S212, N224 and K2261 groups in NCOA1 protein and 1-palmitic acid-2-linoleic acid-3-glyceryl oleate (PLO)(Figure 4B). There are hydrogen bond interactions between K220 and N224 groups in NCOA1 protein and Myricanone (Figure 4C), nCOA2 protein groups A475 and R655 have hydrogen bond interaction with Chrysophanol (Figure 4D), and NCOA2 protein groups D467 and P547 have hydrogen bond interaction with Chuanxiong naphtholide (wallichilide)( Figure 4F).NCOA2 protein N466 group has hydrogen bond interaction with Angelica lactone a (Levistolide-A/Levistolid a)( Figure 4G), and the NCOA2 protein D590 group has hydrogen bond interaction with 1-palmitic acid-2linoleic acid-3-glyceryl oleate (PLO)( Figure 4H).

\section{Discussion}

According to epidemiological data released by 2021,451 million adults aged 20 to 79 worldwide have diabetes, of whom 116 million in China have diabetes [14], in Western countries, 30\%-50\% of people with diabetes have secondary kidney disease [15]. Chronic kidney disease caused by diabetes, called DN, has a large base of patients, and in developed countries, DN has been the main cause of the end-stage renal disease (ESRD) for a long time, and the prognosis is very limmited[16]. At present, western drugs are mainly used in clinical practice, such as ACEI/ARB, but the effect of the simple application is not good. Clinical studies have shown that the combination of Chinese drugs and western drugs can play a better role in the treatment of DN; for example, chuanxiong injection combined with Puli (ACEI) can improve renal function and reduce the level of urine protein better than alone injection[17]. However, although Chinese medicine has a favourable curative effect, it works in the form of multi-component coexistence. Most of the drugs have the problem of unclear specific effective components. Therefore, we seek the main active components of Chinese medicine, and it is necessary to improve the efficiency of drug utilization. 
In recent years, the development of Internet pharmacology has promoted the discovery and utilization of the effective components of traditional Chinese medicine and saved the cost of drug research and development to some extent. Based on the method of network pharmacology, the active components of chuanxiong Hort were selected by Lipinski five rules[13,18] and drug similarity [19]. There are angelica lactone A, ligusticum chuanxiong hort. Naphthalene, Myricetin respectively, 1-palmitic acid-2-linoleic acid3-olein, conifer ferulate, 1-aldehyde- $\beta$-carboline, ligusticum chuanxiong and Chrysophanol; protein interaction analysis using a STRING database in the exploration of the interaction network of core genes in the treatment of DN by chuanxiong, Cytoscape 3.7.2 software was used to optimize the results. The results showed that there was an interaction network between the active components of chuanxiong and DN, in which NR3C1, AR, ESR1, NCOA1, NCOA2 and PGR played a key role in the regulatory network, after GO analysis and KEGG analysis of 15 potential target genes of chuanxiong Hort. regulating DN, the GO results suggest that the genes involved in the regulation of DN by chuanxiong Hort. It is involved in the regulation of cellular response to steroid hormone stimulation, the binding of nuclear receptors to steroid hormone receptors and the regulation of cAMP-dependent protein kinase complexes. Besides, KEGG analysis showed that estrogen signalling pathway, endocrine and other factors-regulated calcium reabsorption and adipocyte adiposis regulation play a role in the regulation of $\mathrm{DN}$. Thus, to find out the sites of interaction and the forms of interaction between the active components and the targets, the key genes and the main active components of chuanxiong hort. After determining the range of the active pocket, we used vina software to predict the molecular docking and select the appropriate binding energy; it was found that the K220, S212, N224, and K2261 groups in NCOA1 protein could interact with the hydrogen bonds of angelica lactone A, palmitic acid-2-linoleic acid-3-glyceryl oleate and Myricetin, the groups of NCOA2 protein A475, R655, D590, N466, D467 and P547 interact with Chrysophanol, chuanxiong naphthafuronolactone, Angelica Sinensis A, 1-palmitic acid-2-linoleic acid-3-glyceryl ester by a hydrogen bond. The above results suggest that there are potential targets of active components of chuanxiong Hort. NCOA1 and NCOA2 have intermolecular interactions with the main active components of chuanxiong Hort.

Clinical and animal studies have shown a link between ESR1 signals activity and DN [20]. Studies have shown that plasma estradiol levels in the kidneys of diabetic patients are reduced, and ESR1 signals are out of whack [21]. Protein tyrosine kinase (PTK) is a kind of kinase that catalyzes the transfer of $\mathrm{Y}^{-}$ phosphoric acid from ATP to protein tyrosine residues [22], and can catalyze the phosphorylation of tyrosine residues in a variety of substrate proteins, plays an important role in cell growth, proliferation and differentiation [23]. PTK can be divided into non-receptor type and membrane receptor type according to whether PTK exists in the cell membrane receptor. The P160/steroid receptor coactivators (SRC-1, SRC-2, also known as NCOA1, NCOA2) family products represent the non-receptor type [24, 25], which can regulate a variety of physiological reactions and clinical pathology. This pleiotropy is achieved through its inherent structural complexity, which enables this class of auxiliary regulators to control both nuclear and nonnuclear receptor signals, playing an important role in steroid hormone signaling [26]. The results indicated that the target genes of chuanxiong Hort. can be regulated by CAMP-dependent protein kinase complexes. It is well known that cAMP can activate the expression of Phosphatidylinositol 3-hydroxy 
kinase (PI3K)[27], it has been suggested that Estrogen can activate PI3K by binding to the ESR1(ER) on the cell membrane and participate in the regulation of Estrogen signalling pathway, which suggests a potential link between the therapy of chuanxiong and the activation of Estrogen signaling[28]. Recent studies have shown that the estrogen signal pathway can affect energy metabolism; KEGG analysis results show that the estrogen signal pathway is involved in the regulation of DN by chuanxiong. Based on the above results, we hypothesized that chuanxiong might participate in the binding of steroid hormone receptors via NCOA1 and NCOA2, thus affecting the activation of an estrogen signalling pathway, regulating energy metabolism and delaying the progression of DN. In this study, the method of network pharmacology was used to systematically analyze the treatment of DN with chuanxiong Hort.

Based on the published literature and the results of molecular docking experiments, we hypothesized that chuanxiong might be involved in the binding of steroid hormone receptors via NCOA1 and NCOA2; it can affect the activation of the estrogen signal pathway, regulate the energy metabolism and delay the progress of DN. It provides a new way for the better clinical application and mechanism research of chuanxiong, and a new method and a new way for the research of traditional Chinese medicine. In this study, the mechanism of action of chuanxiong in the treatment of DN was predicted based on network pharmacological reasoning, and the drug-target interaction was analyzed by molecular docking technique in order to improve the reliability of the target of chuanxiong Hort., the prediction results need to be verified by in vitro and in vivo experiments.

\section{Declarations}

\section{Author Contributions}

All authors contributed to developing the guideline, the planning, drifting, and revision of this summary approved the final version and takes responsibility for it.

\section{Acknowledgements}

This work was supported by a grant from National Natural Science Foundation of China (Project Number: 82160142), Yunnan Health Technical Training Project of High-level Talents (Project Number: D-2018027) and Yunnan Basic Research Projects - General Program (Project Number: 2019FB092).

\section{Disclosure}

All authors of this study are responsible for the article's accuracy, have no doubts about the byline, and without conflict of interest.

\section{References}

1. Z.F. Zhou, L. Jiang, Q. Zhao, et al. "Roles of pattern recognition receptors in diabetic nephropathy," $J$ Zhejiang Univ Sci B, vol. 21, no. 3, pp. 192-203. 
2. T.P. Griffin, P.M. O'Shea, A. Smyth, et al. "Burden of chronic kidney disease and rapid decline in renal function among adults attending a hospital-based diabetes center in Northern Europe," BMJ Open Diabetes Research and Care, vol. 9, no. 1, p. e002125.

3. H. Zhang, S. Qi, Y. Song, and C. Ling. "Artemisinin attenuates early renal damage on diabetic nephropathy rats through suppressing TGF- $\beta 1$ regulator and activating the Nrf2 signaling pathway," Life sciences, vol. 256, p. 117966.

4. D. Sharma, R. Kumar Tekade, and K. Kalia. "Kaempferol in ameliorating diabetes-induced fibrosis and renal damage: An in vitro and in vivo study in diabetic nephropathy mice model," Phytomedicine: international journal of phytotherapy and phytopharmacology, vol. 76, p. 153235.

5. J. ALTamimi, N. AlFaris, A. Al-Farga, G. Alshammari, M. BinMowyna, and M. Yahya. "Curcumin reverses diabetic nephropathy in streptozotocin-induced diabetes in rats by inhibition of PKC $3 / p S h c$ axis and activation of FOXO-3a," The Journal of nutritional biochemistry, vol. 87, p. 108515.

6. F. Wang, R. Li, L. Zhao, S. Ma, and G. Qin. "Resveratrol ameliorates renal damage by inhibiting oxidative stress-mediated apoptosis of podocytes in diabetic nephropathy," European journal of pharmacology, vol. 885, p. 173387.

7. C. Zuo and G. Xu. "Efficacy and safety of mineralocorticoid receptor antagonists with ACEI/ARB treatment for diabetic nephropathy: A meta-analysis," International journal of clinical practice, $\mathrm{p}$. e13413.

8. K. Umanath and J. Lewis. "Update on Diabetic Nephropathy: Core Curriculum 2018," American journal of kidney diseases : the official journal of the National Kidney Foundation, vol. 71, no. 6, pp. 884-895.

9. S. Hu, S. Chen, Z. Li, Y. Wang, and Y. Wang. "Research on the potential mechanism of Chuanxiong Rhizoma on treating Diabetic Nephropathy based on network pharmacology," International journal of medical sciences, vol. 17, no. 15, pp. 2240-2247.

10. X. Yuan, B. Han, Z. Feng, J. Jiang, Y. Yang, and P. Zhang. "Chemical constituents of Ligusticum chuanxiong and their anti-inflammation and hepatoprotective activities," Bioorganic chemistry, vol. 101, p. 104016.

11. S. Zhang, L. An, Z. Li, et al. "Structural elucidation of an immunological arabinan from the rhizomes of Ligusticum chuanxiong, a traditional Chinese medicine," International journal of biological macromolecules, vol. 170, pp. 42-52.

12. Y. Song and D.O. Nephrology. "Randomized Controlled Study of Chuanxiong Injection combined with Benazepril in the Treatment of Diabetic Nephropathy," Journal of Practical Traditional Chinese Internal Medicine.

13. X. Chen, H. Li, L. Tian, Q. Li, J. Luo, and Y. Zhang. "Analysis of the Physicochemical Properties of Acaricides Based on Lipinski's Rule of Five," Journal of computational biology : a journal of computational molecular cell biology, vol. 27, no. 9, pp. 1397-1406.

14. R. Williams, S. Karuranga, B. Malanda, et al. "Global and regional estimates and projections of diabetes-related health expenditure: Results from the International Diabetes Federation Diabetes Atlas, 9th edition," Diabetes research and clinical practice, vol. 162, p. 108072. 
15. Y. Deng, N. Li, Y. Wu, et al. "Global, Regional, and National Burden of Diabetes-Related Chronic Kidney Disease From 1990 to 2019," Frontiers in endocrinology, vol. 12, p. 672350.

16. H. Cheng, X. Xu, P. Lim, and K. Hung. "Worldwide Epidemiology of Diabetes-Related End-Stage Renal Disease, 2000-2015," Diabetes care, vol. 44, no. 1, pp. 89-97.

17. "Exploration of the Compatibility of Ligusticum Wallichii in the Prevention and Treatment of Diabetic Nephropathy," China Journal of Chinese Medicine.

18. "Going further than Lipinski's rule in drug design," Expert Opinion on Drug Discovery.

19. "DTiGEMS+: Drug-target interaction prediction using graph embedding, graph mining, and similaritybased techniques," Journal of Cheminformatics, vol. 12, no. 1.

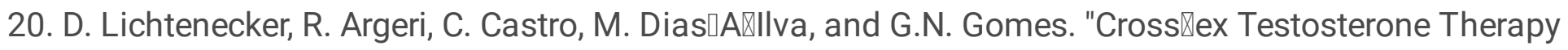
Modifies the Renal Morphology and Function in Female Rats and Might Underlie Increased Systolic Pressure," Clinical and Experimental Pharmacology and Physiology.

21. "Quercetin promotes long noncoding RNA IncSHGL expression via estrogen receptor a to suppress type 2 diabetes mellitus in mice."

22. "Recent advances in pharmacological diversification of Src family kinase inhibitors," Egyptian Journal of Medical Human Genetics, vol. 22, no. 1.

23. E.J.M. van Bommel, M.H.A. Muskiet, M.J.B. van Baar, et al. "The renal hemodynamic effects of the SGLT2 inhibitor dapagliflozin are caused by post-glomerular vasodilatation rather than preglomerular vasoconstriction in metformin-treated patients with type 2 diabetes in the randomized, double-blind RED trial," Kidney Int, vol. 97, no. 1, pp. 202-212.

24. V.C. Cheung, C.Y. Peng, M. Marini, et al. "Pluripotent stem cell-derived endometrial stromal fibroblasts in a cyclic, hormone-responsive, coculture model of human decidua - ScienceDirect."

25. H. Jheng, K. Hayashi, Y. Matsumura, T. Kawada, and T. Goto. "Anti囚nflammatory and Antioxidative Properties of Isoflavones Provide Renal Protective Effects Distinct from Those of Dietary Soy Proteins Against Diabetic Nephropathy," Molecular Nutrition \& Food Research, vol. 64, no. 10.

26. A. Kas, A. Bs, C. Kd, C. Dk, C. Dk, and C. Rlb. "4-thiazolidinone-based derivatives rosiglitazone and pioglitazone affect the expression of antioxidant enzymes in different human cell lines," Biomedicine \& Pharmacotherapy, vol. 139.

27. G. Saw, K. Krishna, N. Gupta, T. Soong, and S.T. Dheen. "Epigenetic regulation of microglial phosphatidylinositol 3区inase pathway involved in long-term potentiation and synaptic plasticity in rats," Glia, vol. 68, no. 6.

28. "Astragali Radix Isoflavones Synergistically Alleviate Cerebral Ischemia and Reperfusion Injury Via Activating Estrogen Receptor-PI3K-Akt Signaling Pathway," Frontiers in Pharmacology, vol. 12.

\section{Figures}


A
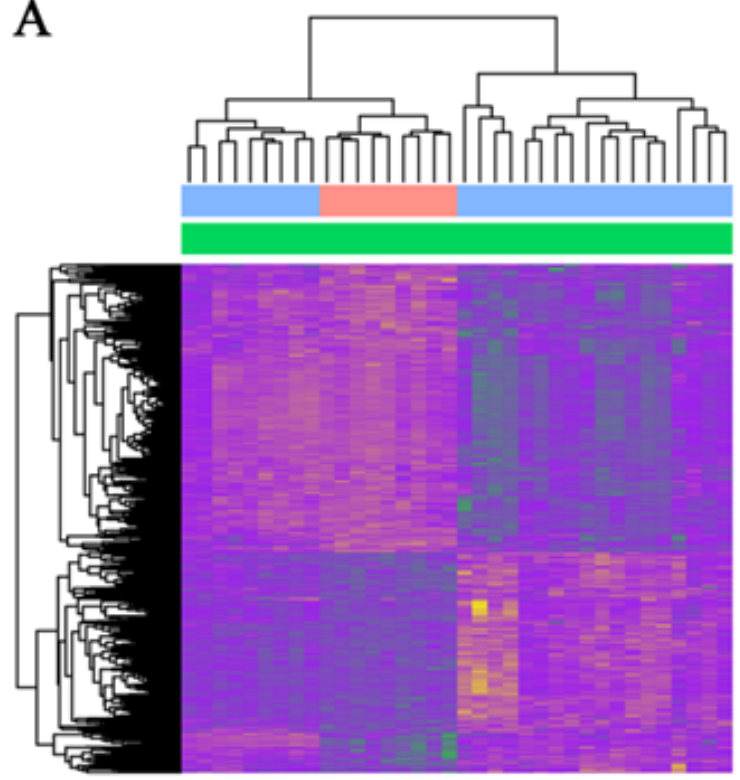

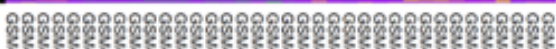

A

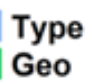

Type

4 con
DN

2

Geo

0

$-2$

$-4$

D

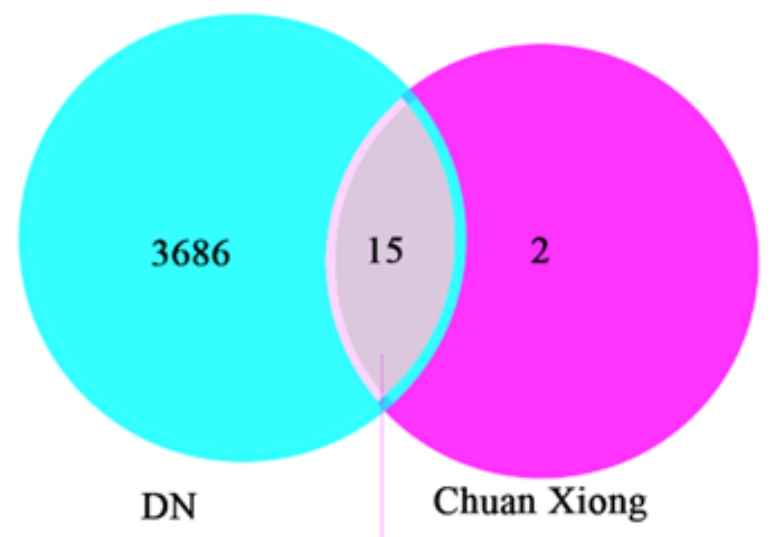

PGR NR3C1 NCOA1 NCOA2 NR3C2 PTGS1

F2 ESR1 AR PPARG F7

ESR2 DPP4 HSP90AA1 PRKACA
B
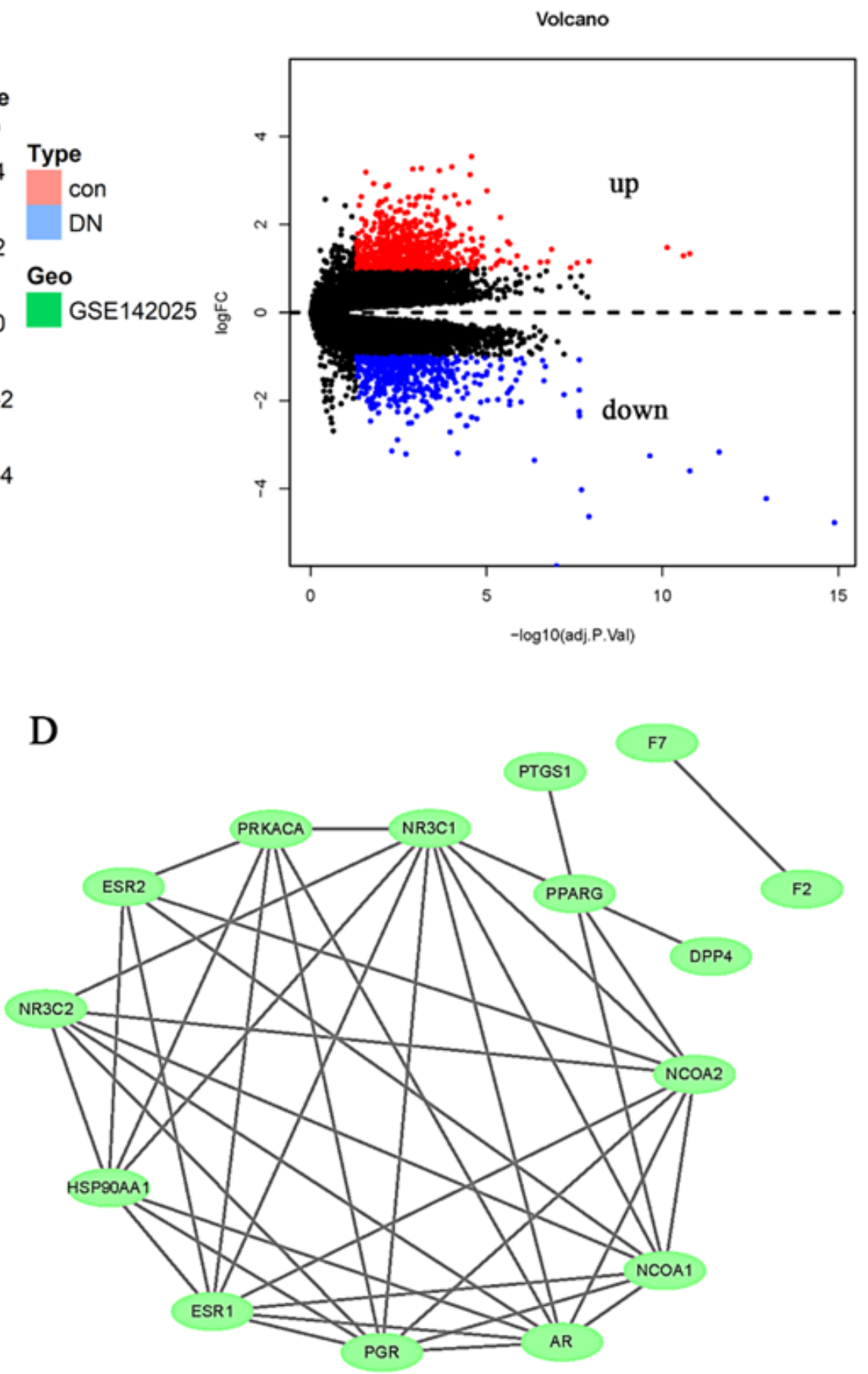

Figure 1

Analysis of the interaction between a target of active components of chuanxiong Hort and DN differential genes A, B: Differential Thermogram and volcano map of DN dataset; C: Differential Gene and Veen analysis of chuanxiong Target; D: PPI interaction analysis of target gene. 
A

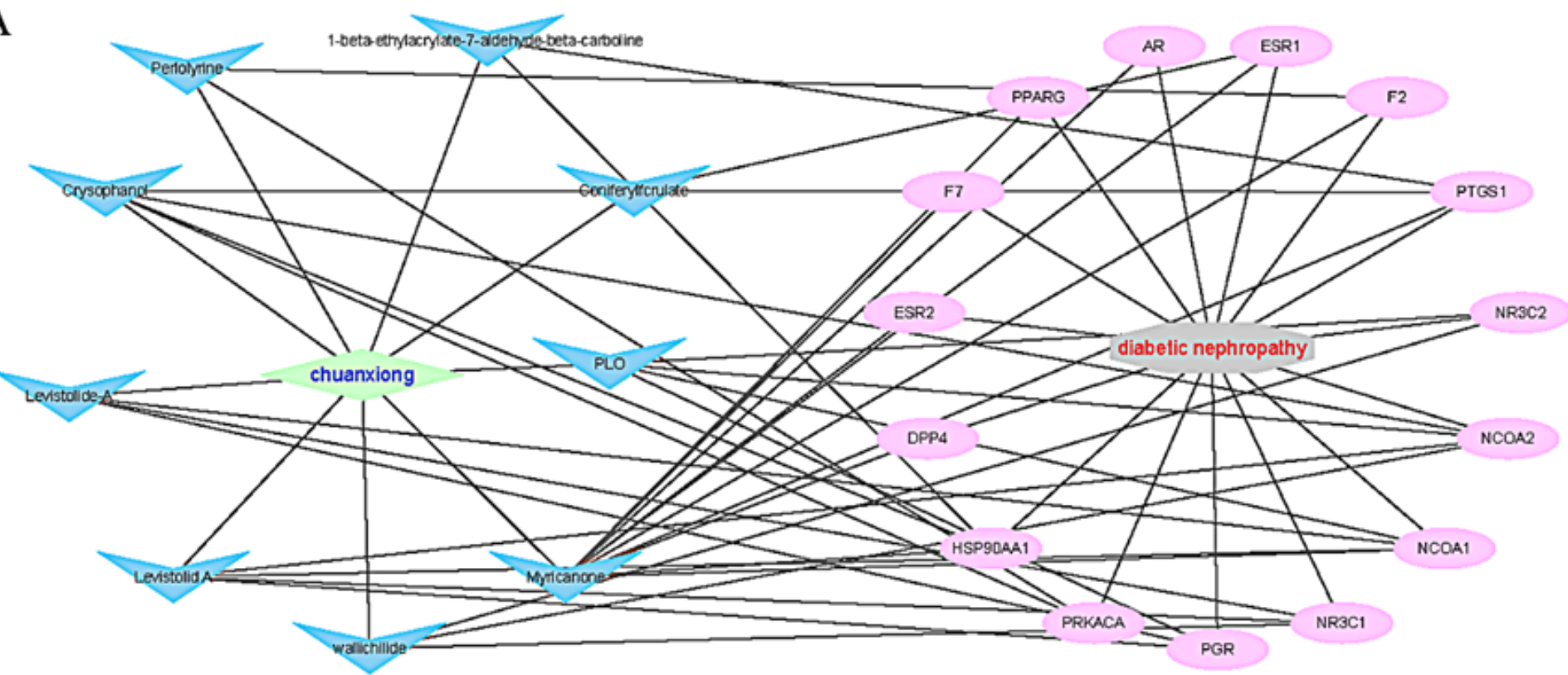

B

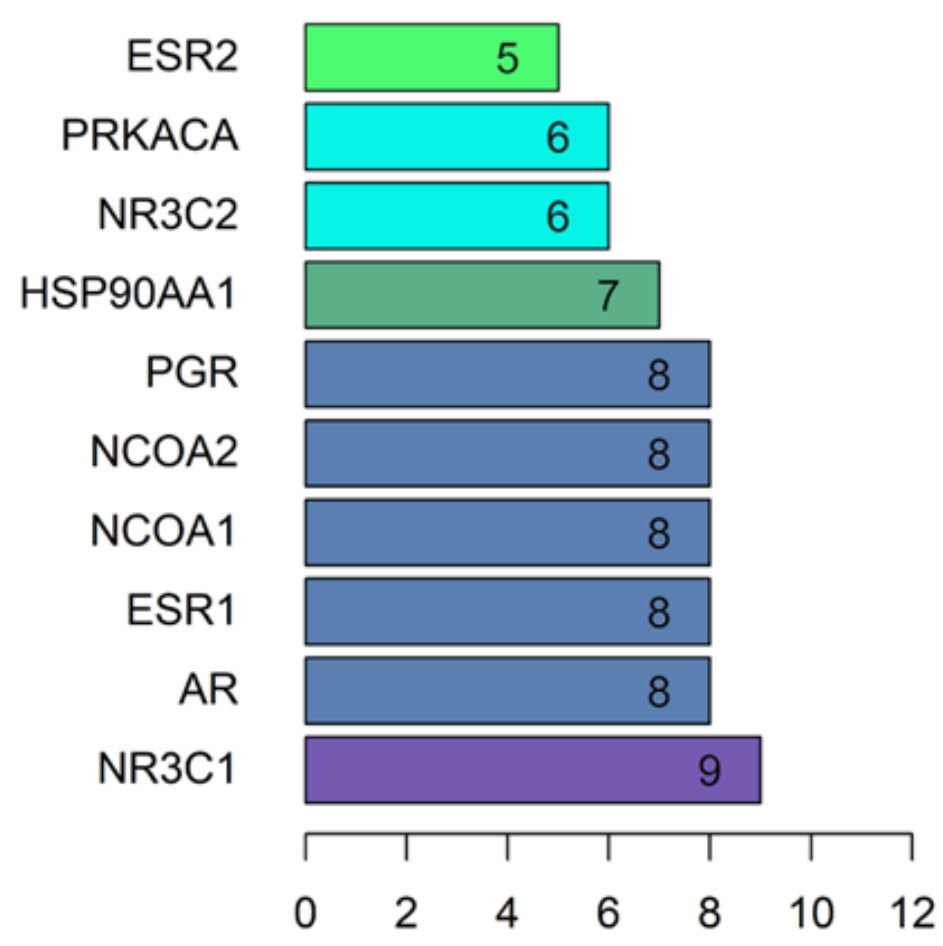

Figure 2

Regulatory network of active ingredients of chuanxiong Hort. in the treatment of DN. A: the interaction network of DNA regulated by active components of chuanxiong Hort. B: the frequency of gene interaction in the network. 


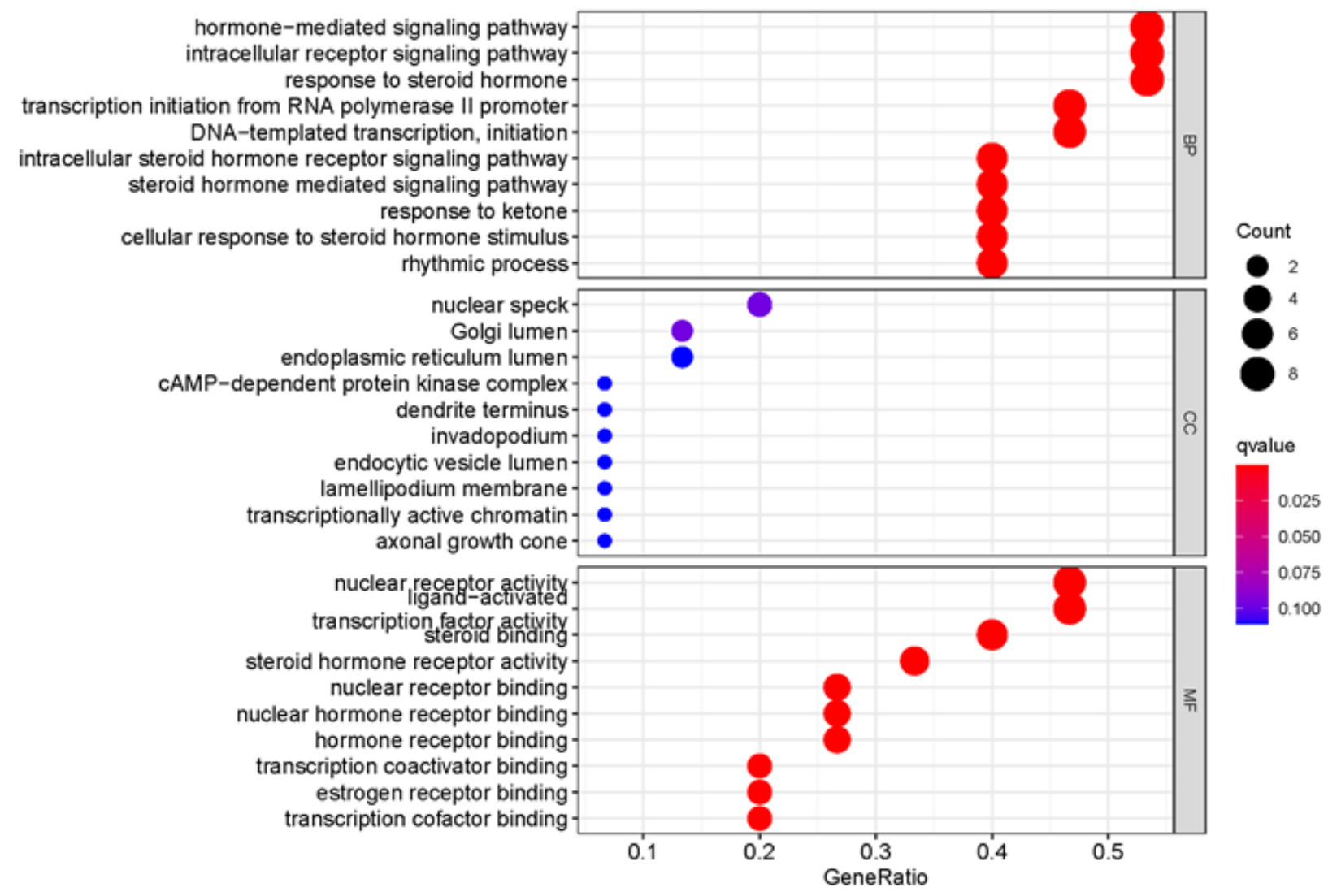

B

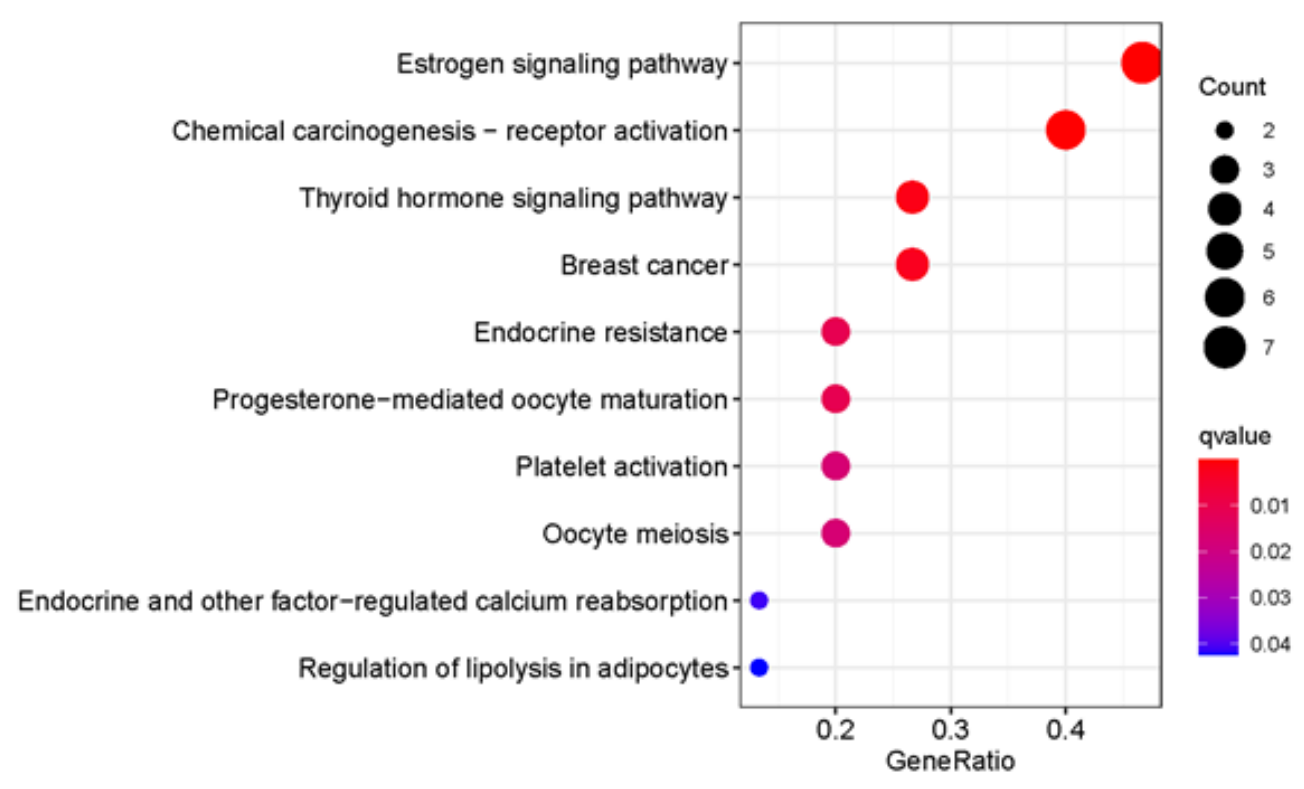

\section{Figure 3}

Enrichment and analysis of $\mathrm{GO}$ and KEGG, the targets of regulating DN by active components of chuanxiong Hort. A: enrichment analysis of GO, a potential target gene of chuanxiong regulating DN; $\mathrm{B}$ : Enrichment Analysis of KEGG, a potential target gene of chuanxiong regulating DN. 


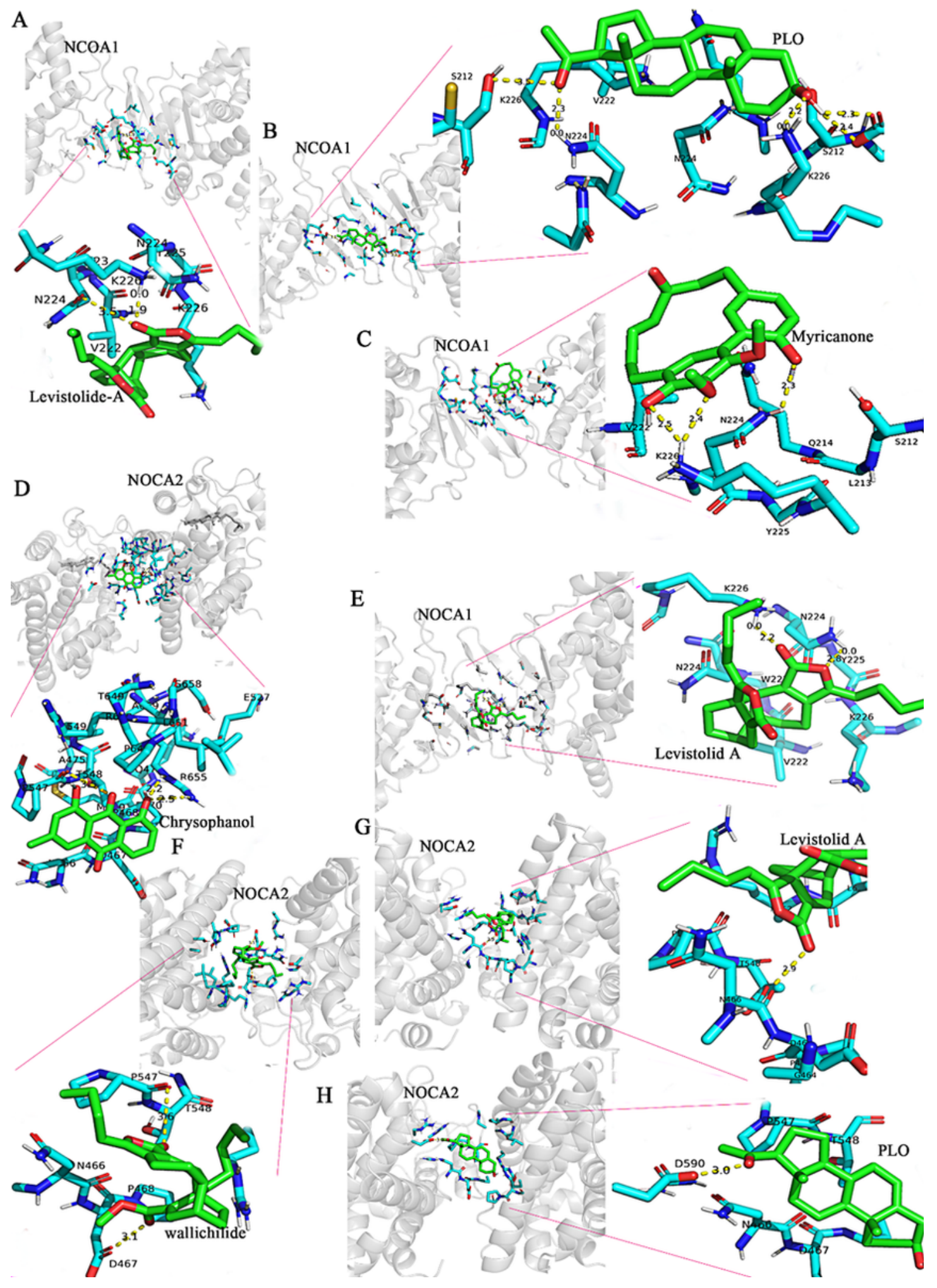

Figure 4

Molecular docking analysis of NCOA1 and NCOA2 proteins with active components of chuanxiong Hort. A, B, C, E: the groups in NCOA1 protein interacted with a (Levistolide-A/Levistolid A, Palmitic acid-2linoleic acid-3-glyceryl ester (PLO) and Myricanone; D, F, G, H: the groups in NCOA2 protein interacted with Chrysophanol, wallichilide, Angelica lactone a (Levistolid-A/Levistolid A) and 1-palmitic acid-2-linoleic acid-3-glyceryl ester (PLO). 